\title{
Corrosión atmosférica acelerada en una fundición gris ASTM A48
}

\section{Accelerated atmospheric corrosion in a cast ASTM A48 gray iron}

\author{
Alfredo Artigas Abuin ${ }^{1,2}$, Rodrigo Allende Seco ${ }^{1}$, Alberto Monsalve González ${ }^{1,2}$, \\ Konstantin Sipos Hrabal $^{1}$, Ariel Soto Coña ${ }^{1}$
}

\begin{abstract}
${ }^{1}$ Departamento de Ingeniería Metalúrgica, Facultad de Ingeniería, Universidad de Santiago de Chile, Av. L. B. O’Higgins 3363, Estación Central, Santiago, Chile. CP 9160000. e-mail: alfredo.artigas@usach.cl, rodrigo.seco@usach.cl, alberto.monsalve@usach.cl,ksiposh@yahoo.com, ariel.sotoc@usach.cl

${ }^{2}$ Laboratorio SIMET-USACH del Departamento de Ingeniería Metalúrgica, Facultad de Ingeniería, Universidad de Santiago de Chile, Av. L. B. O’Higgins 3363, Estación Central, Santiago, Chile. CP 9160000.
\end{abstract}

\section{RESUMEN}

Debido a los extensos tiempos que conllevan los estudios relativos a resistencia a la corrosión atmosférica, estos datos son escasos. Uno de estos, es el realizado por Southwell et al., en el que se estudió el efecto de la corrosión durante 16 años para una fundición gris en una diversidad de ambientes.

El presente trabajo expone y discute los resultados obtenidos tras someter a una fundición gris a un ensayo de corrosión acelerada, que involucra ciclos de humectación en cámara de niebla, lavado y secado, durante 40 días. Además, se ha realizado una experiencia en la que se sumergieron muestras de fundición gris en agua de mar natural y agua de mar esterilizada con UV, encontrando una relación directa entre la presencia de bacterias y un aumento en la rugosidad superficial de las muestras expuestas a estas soluciones durante 14 días.

Los resultados obtenidos revelaron que la fundición gris presenta un comportamiento bimodal, que es concordante con lo descrito por Melchers para aceros de baja aleación y con la investigación desarrollada por Southwell et al. Este comportamiento bimodal, según Melchers et al. genera un punto de inflexión en el avance de la corrosión, influenciado por bacterias en la interfase metal-productos de corrosión. Esto último, se valida evidenciando actividad bacteriana tras identificar presencia de Azufre, mediante EDS, en los productos de corrosión desarrollados tras la exposición al ensayo de corrosión acelerada.

Finalmente se puede comentar que en pocas semanas y empleando un ensayo de corrosión acelerada es posible replicar las condiciones bajo las que se realiza corrosión atmosférica marina durante años, para una fundición gris, incluso evidenciando actividad bacteriana.

Palabras clave: corrosión atmosférica, fundición gris, pérdida en peso, corrosión bacteriana.

\section{ABSTRACT}

Due to the long times required to investigate atmospheric corrosion resistance of metals, data on the subject is scarce. One of these studies is the one made by Southwell et al., where the effect of corrosion in a cast gray iron during 16 years was studied in various environments.

This research shows and discusses the behavior of cast gray iron when subject to an accelerated corrosion test, which involves wetting in a salt spray chamber, washing and drying cycles during 40 days. Furthermore, samples were submerged in sea water, both natural and UV-sterilized. A direct relationship amongst the presence of bacteria and an increase of surface roughness was found when subject to these conditions during 14 days.

Results show that the cast gray iron shows a bimodal behavior, like the observations reported by Melchers on low-alloy steels and the research by Southwell.et al. As described by Melchers, this behavior generates an inflection point in the evolution of corrosion influenced by the presence of bacteria on the metal/corrosionproducts interphase. This is validated observing evidence of bacterial activity identifying the presence of sulphur on the corrosion products by EDS after the accelerated corrosion tests. 
As observed, accelerated corrosion testing can simulate in a short period the conditions under which marine atmospheric corrosion takes place on cast gray iron during years, including the evidence of bacterial activity.

Keywords: atmospheric corrosion, gray cast iron, weight loss, bacterial corrosion.

\section{INTRODUCCIÓN}

En la actualidad existen pocos estudios sobre la resistencia a la corrosión atmosférica de los materiales en general y en particular de las aleaciones ferrosas. Algunos de ellos se han concentrado en caracterizar ambientes de corrosión [1] y otros en evaluar la resistencia a la corrosión atmosférica marina en aceros [2,3] y fundiciones [4-6]. La realización de trabajos como estos tiene una desventaja en común y es que para su realización se requiere de años, por ello algunos autores y en particular este equipo de trabajo, se han concentrado en buscar la forma de reducir estos tiempos a través de la realización de experiencias de corrosión acelerada [7-16], que tras poco tiempo (días) y en laboratorio puedan generar resultados concordantes con los obtenidos en años de exposición a la intemperie.

Continuando con la búsqueda de validar una metodología para la reducción de tiempos de investigación de la resistencia a la corrosión atmosférica marina de los materiales, es que este trabajo tiene por objetivo obtener resultados de espesor corroído en el tiempo para una fundición gris A48 [17], para luego comparar estos resultados de laboratorio, obtenidos mediante un ensayo de corrosión acelerada [7], con los obtenidos tras años de exposición a la intemperie [4].

\subsection{Modelos de corrosión}

Como primera aproximación a la determinación de corrosión en el largo plazo, se han utilizado modelos del tipo potencial, como el presentado en la Ecuación 1, donde C representa el espesor corroído, t el tiempo de exposición y A y B son constantes. La forma de esta expresión surge del supuesto que la corrosión comienza inmediatamente y se produce de manera indefinida $[18,19]$. No obstante, existen ciertos productos de corrosión que protegen los materiales frente a este fenómeno y por tanto la concentración de oxígeno sobre el sustrato es distinta en el tiempo.

Con el propósito de considerar los elementos anteriormente descritos y que quedan excluidos en la determinación de la Ecuación 1, es que Melchers [20] propone que la corrosión en aleaciones ferrosas, es un fenómeno que cambia en el tiempo, identificándose dos fases; la primera, en que la corrosión es aeróbica y la segunda, anaeróbica influenciada principalmente por la actividad de bacterias reductoras de sulfato. Se encuentra así una relación entre espesor corroído y tiempo de exposición a la intemperie, del tipo bi-modal, como la ilustrada en la Figura 1.

$$
C=A t^{B}
$$

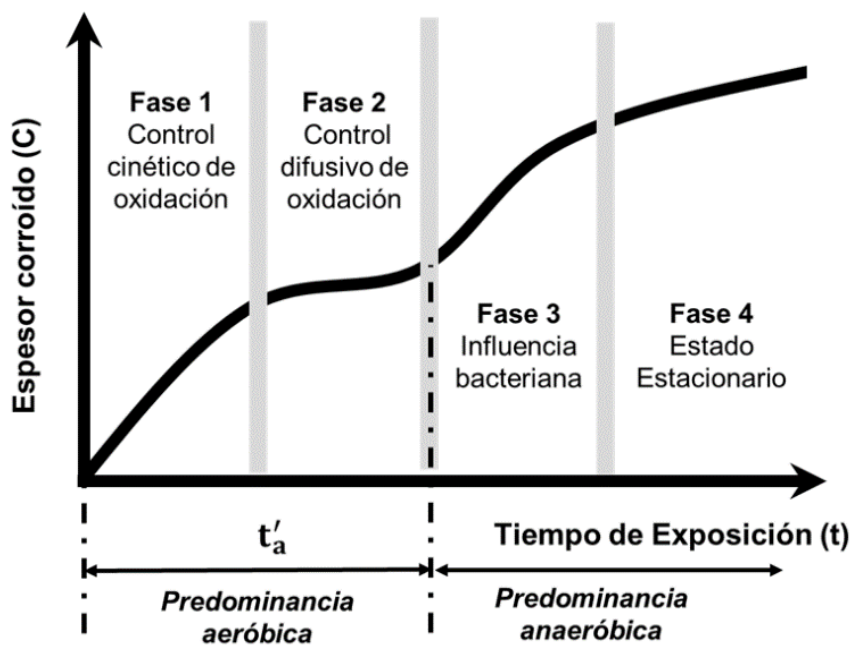

Figura 1: Esquema del modelo bi-modal para espesor corroído, mostrando sus principales fases.

El término $t_{a}^{\prime}$ marca la transición de una predominancia a otra y de acuerdo a observaciones de datos experimentales [21] se alcanza durante los primeros años de exposición a la intemperie (3 a 6 años), por ello durante la realización de ensayos de corrosión acelerada se pondrá énfasis en los primeros ciclos. 


\section{MATERIALES Y MÉTODOS}

A continuación, se presentarán las cuatro partes en que se dividen las experiencias y metodología utilizada.

\subsection{Ciclos de corrosión acelerada}

Para desarrollar esta etapa, se prepararon cupones de $50 \times 30 \times 6$, todas en mm, de la fundición gris (FG) y acero estructural (AE). Estos cupones una vez medidos y pesados fueron incorporados a los ciclos de corrosión acelerada de 24 horas de duración cada uno. Cada ciclo consta de las siguientes etapas, con un total de 24 (h) por ciclo:

1. 3 (h) en cámara de niebla salina, según la norma ASTM B117 [22].

2. 2 (h) de secado

3. 17 (h) en cámara de niebla salina

4. Lavado de las muestras con una ducha

5. 2 (h) de secado

La etapa de lavado, cumple un rol fundamental en la experiencia, ya que por un lado simula la erosión ambiental que experimenta el acero y por otro lado baja de manera considerable las concentraciones de $\mathrm{NaCl}$, lo cual permite la formación de la pátina protectora.

Las muestras estuvieron sometidas durante 40 ciclos (960 (h)), extrayendo muestras en los ciclos 3, 5, $6,9,10,12,15,20,25,30,35$ y 40 (se ha puesto el foco en los primeros ciclos, de acuerdo a lo comentado con anterioridad).

\subsection{Análisis morfológico de productos de corrosión}

Con la finalidad de evaluar la morfología, la compactación y grietas de los productos de corrosión presentes en los cupones, se realizó microscopía electrónica de barrido (SEM). Ya en un trabajo anterior del grupo de investigación [7], se demostró que empleando el ensayo de corrosión acelerada descrito en la sección 2.1, se pueden obtener en pocas semanas los productos de corrosión reportados en la literatura tras varios años de exposición a la intemperie [23], los resultados de este estudio [7], se identificaron, cuantificaron los productos de corrosión (Goethita, Lepidocrocita; Akaganeita, Hematita, Maghemita/Magnetita) que son concordantes con la corrosión a largo plazo, expresada en la literatura. Si bien es cierto que los productos de corrosión formados en la fundición gris no fueron analizados, es esperable que presente este mismo tipo de oxihidróxidos, tal como dice la literatura. [24,25], no siendo relevantes para este estudio, pues lo que se desea replicar es el comportamiento bimodal del material frente a la corrosión atmosférica marina.

Para mantener adheridos al cupón los productos de corrosión, se pegó a éste un film de aluminio con resina epóxica. Posteriormente se realizó un corte transversal con un disco de corte refrigerado. Una vez cortado, el cupón recubierto fue montado en baquelita conductora para microscopía electrónica. Luego, los cupones montados fueron lijados, pulidos y luego atacados químicamente empleando una solución de ácido clorhídrico al 33\% durante 5 (s). Se realizó este procedimiento para muestras obtenidas tras el ciclo 10, 20 y 40.

\subsection{Determinación de espesores corroídos}

El espesor corroído promedio se calculó a partir de la pérdida de peso (método gravimétrico) y las velocidades de corrosión mediante la Ecuación 2, tal como lo especifica la norma ASTM G1 [26].

$$
V=\frac{K W}{D A T}
$$

$V$ es la velocidad de corrosión, $K$ es una constante que depende de las unidades

finales de la velocidad de corrosión y de las unidades usadas en el resto de los parámetros, $W$ es la pérdida de peso, $D$ es la densidad de la muestra, $A$ es el área total y $T$ el tiempo de exposición. Se emplearon duplicados en cada uno de los ciclos estudiados. 


\subsection{Estudio de influencia bacteriana}

En esta sección lo que se pretendió fue establecer una relación entre la presencia de bacterias y la corrosión localizada en la fundición gris. Para lo anterior, se procedió a sumergir cupones de fundición gris, en dos tipos de agua por separado. El primero, agua de mar natural de las costas de Antofagasta Chile; y el segundo, esta misma solución, pero expuesta a radiación UV-C. Esta segunda solución corresponde a una que presenta ausencia de bacterias, de acuerdo al efecto bactericida de los rayos UV-C [27]. Tras 14 (días) de exposición a las soluciones las muestras fueron retiradas, limpiadas y se realizó una inspección con electrones secundarios en SEM.

\section{RESULTADOS Y DISCUSIONES}

\subsection{Caracterización de material}

Las composiciones químicas de las fundiciones estudiadas, tanto en la intemperie, en el Canal de Panamá, como el realizado en este trabajo para el ensayo de corrosión acelerada, se presentan en la Tabla 1.

La fundición gris sometida a corrosión acelerada presenta una microestructura típica para este tipo de materiales, siendo una matriz ferrítico-perlítica con presencia de grafito laminar distribuido de forma homogénea.

Tabla 1: Composiciones químicas Fundiciones

\begin{tabular}{||c|c|c|c|c|c|c|c|c||}
\hline $\begin{array}{c}\text { Muestra } \\
\text { ID }\end{array}$ & $\% \mathbf{C}$ & $\% \mathbf{M n}$ & $\% \mathbf{P}$ & $\% \mathbf{S}$ & $\% \mathbf{S i}$ & $\% \mathbf{C r}$ & $\% \mathbf{N i}$ & $\% \mathbf{C u}$ \\
\hline \hline $\begin{array}{c}\text { FG } \\
\text { Canal de } \\
\text { Panamá [4] }\end{array}$ & 3,19 & 0,80 & 0,16 & 0,10 & 1,98 & 0,57 & 0,31 & 0,08 \\
\hline \hline $\begin{array}{c}\text { FG } \\
\text { Corrosión } \\
\text { Acelerada }\end{array}$ & 3,43 & 0,90 & 0,02 & 0,02 & 2,68 & 0,02 & - & 0,46 \\
\hline
\end{tabular}

\subsection{Análisis de resultados posterior a ensayo de corrosión acelerada}

La tabla 2 presenta los valores promedio de pérdida en peso (en g) por unidad de área superficial (en $\mathrm{m}^{2}$ ) asociadas al ensayo de corrosión acelerada, con estos resultados y considerando una densidad del acero de 7,81 $\mathrm{g} / \mathrm{cm}^{3}$, se obtuvieron los espesores corroídos en el tiempo.

La Figura 2 presenta, a la izquierda, la curva de espesores corroídos en función del tiempo de ensayo de corrosión acelerada, luego de 40 ciclos (días) y a la derecha, los espesores corroídos obtenidos por Southwell et. al [4] para una fundición gris en el canal de Panamá, tras 16 años de exposición a la intemperie en un ambiente marino cercano a la costa. De la comparación de estas curvas se puede comentar que al igual que en la curva obtenida en años, la curva de espesores corroídos obtenidos a través de un ensayo acelerado, responde a un modelo del tipo bi-modal como el descrito por Melchers [20, 21] o bi-lineal de acuerdo a lo descrito por Albretch y Hall [28]. Los resultados obtenidos presentan un sesgo menor al $5 \%$.

Tabla 2: Pérdidas en peso sobre área superficial asociadas a ensayo de corrosión acelerada.

\begin{tabular}{||c||c|c|c|c|c|c|c|c|c||}
\hline Número de ciclos & 0 & 5 & 10 & 15 & 20 & 25 & 30 & 35 & 40 \\
\hline \hline $\begin{array}{c}\text { pérdida en peso } \\
\text { por unidad de área } \\
\mathbf{g} / \mathbf{m}^{2}\end{array}$ & 0,0 & 369,4 & 623,6 & 687,6 & 710,9 & 744,0 & 787,0 & 817,2 & 836,6 \\
\hline
\end{tabular}





Figura 2: Espesores corroídos para una fundición gris. A la izquierda, corrosión acelerada y a la derecha el ensayo en tiempo real [4].

Existe diferencia de los espesores corroídos en el tiempo, la cual se atribuye a la diferencia de composiciones químicas que presentan ambas fundiciones de estudio, tal como antes se ha discutido para aceros [2, $3,18]$. Sería esperable que el comportamiento fuera similar si las composiciones químicas fueran las mismas [9]. No obstante, ambas curvas presentan un punto de inflexión ( $\mathrm{t}_{\mathrm{a}}$ ) que, de acuerdo a lo descrito en la literatura, marca el punto a partir del cual el fenómeno de corrosión pasa de una predominancia aeróbica a una anaeróbica y bacteriana [21].

De acuerdo a lo presentado en la literatura [21], $\mathrm{t}_{\mathrm{a}}$ se da luego de algunos años de exposición a la intemperie, dependiendo de la concentración de contaminantes ambientales, tiempos de humectación, composición química del sustrato, entre otros. De lo anterior y de la observación de la Figura 2, se puede comentar que tras 12 ciclos de corrosión acelerada (tiempo $t_{a}$ para corrosión acelerada), se obtienen indicios de actividad bacteriana, que a la intemperie se da tras algunos años de exposición, lo que refuerza que el ensayo empleado, en pocas semanas, replica las condiciones bajo las que se realiza la corrosión atmosférica durante años.

Al observar imágenes de SEM, presentadas en la Figura 3, que corresponden a muestras obtenidas luego de los ciclos 10 (antes de $\mathrm{t}_{\mathrm{a}}$ ) y 20 (después de $\mathrm{t}_{\mathrm{a}}$ ) se puede comentar que la morfología de los productos de corrosión pasa de ser una sola fase oxidada porosa (OP) (antes de $\mathrm{t}_{\mathrm{a}}$ ) a una doble capa (después de $\mathrm{t}_{\mathrm{a}}$ ), que exhibe una capa externa (CE) y una interna (CI). El desarrollo de esta doble capa, poco antes de $t_{a}$, indica la presencia de oxihidróxidos de hierro [29] que protegen al sustrato metálico del avance de la corrosión [30] que, al ralentizar la corrosión mediante una disminución en la difusión de oxígeno al sustrato, promueven las condiciones anaeróbicas para la corrosión bacteriana, producto de bacterias reductoras de sulfato (BRS). La acción de estas bacterias sería responsable del picado que aparece luego del punto de inflexión mencionado [31-32].
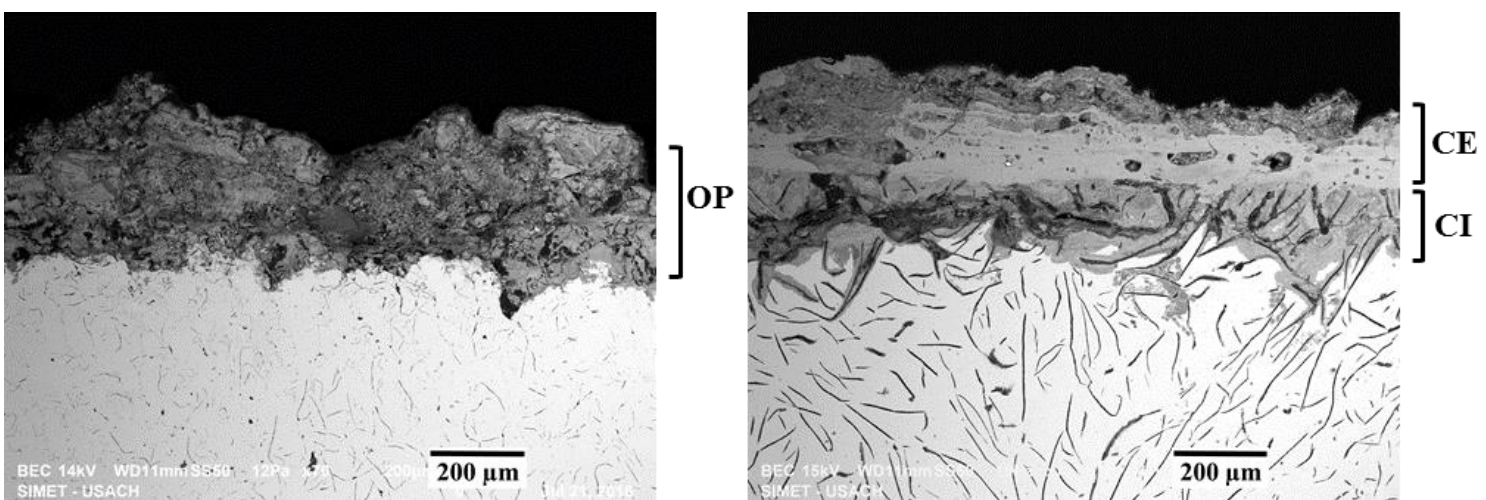

Figura 3: Perfil de productos de corrosión tras exposición a corrosión acelerada. Izquierda, 10 ciclos (antes de $t_{\mathrm{a}}$ ); Derecha, 20 ciclos (después de $t_{a}$ ) 
La presencia de corrosión localizada como responsabilidad de las BRS también fue visualizada en la experiencia en que se sumergieron muestras de fundición gris en agua de mar natural y agua de mar esterilizada con UV. Se ha preferido este método de esterilización con el afán de no modificar las características físicas y químicas del agua de mar empleada. La figura 4 presenta imágenes superficiales tras limpiar cupones expuestos durante 14 días a las aguas mencionadas (se incluye referencia antes de la exposición. Las rugosidades medidas para las muestras sumergidas son.7,69 $\mu \mathrm{m}$ para el caso de agua natural y 6,04 para el caso del agua de mar esterilizada con UV. De lo anterior se deduce que la acción de las bacterias durante la corrosión se realiza de manera localizada (en zonas en las que se dan condiciones anaeróbicas) promoviendo el picado en estas zonas.


Figura 4: Cupones limpiados. (a) antes de la exposición de corrosión, (b) tras 14 días de exposición a agua de mar natural y (c) de agua de mar esterilizada con UV durante 14 días.

De lo anterior, se ratifica que la corrosión localizada es influenciada por la presencia de BRS, resultado concordante con lo expuesto para aceros estructurales. Otro indicio de la influencia de bacteriológica sobre el punto de inflexión encontrado para la fundición gris estudiada bajo corrosión acelerada, es la presencia de azufre que indica el análisis químico puntual mediante EDS, el cual se realizó en la vecindad de una picadura de una muestra, luego del ciclo 40 (Figura 5).

La presencia de azufre se debe a la reducción de sulfato presente en el agua potable con la que se preparó la solución salina, de la sección de humectación salina del ensayo de corrosión acelerada [33]. Adicionalmente, en la Figura 5, se presenta el análisis químico puntual en el seno del producto de corrosión, en el que solo se aprecia la presencia de $\mathrm{Fe}$ y $\mathrm{O}$, puesto que no se darían las condiciones necesarias (anaeróbicas) para la reducción de sulfato por parte de las BRS. 

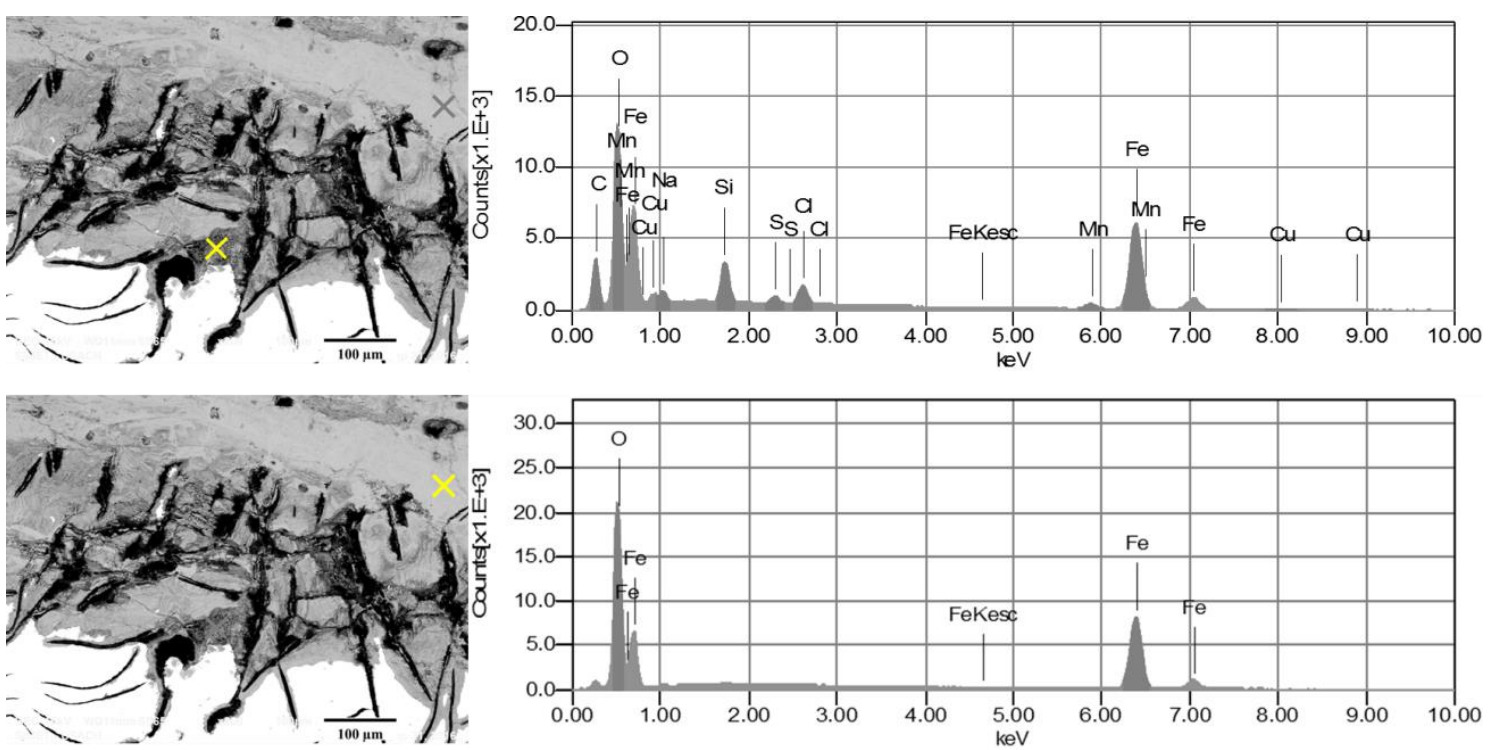

Figura 5: Análisis EDS de fundición gris sometida a 40 ciclos de corrosión acelerada. Arriba, en la vecindad de una picadura. Abajo, en el seno de los productos de corrosión.

De acuerdo a los resultados presentados con antelación, se puede comentar que mediante el uso de un ensayo de corrosión acelerada, durante 40 días, se han podido replicar las condiciones bajo las que se realiza corrosión atmosférica marina para una fundición gris, obteniendo resultados que son concordantes con los presentados en estudios realizados durante 16 años, observando un modelo del tipo bimodal e incluso encontrando evidencia de corrosión anaeróbica bacteriana.

\section{CONCLUSIONES}

Mediante un ensayo de corrosión atmosférica marina simulada se puede predecir el comportamiento en tiempo real de una fundición gris, incluso pudiendo reproducir el comportamiento bimodal y corrosión del tipo bacteriana de aleaciones ferrosas. Si se estableciera una relación entre tiempo de ensayo y tiempo real de exposición a la intemperie en ambiente marino, se podrían analizar nuevas aleaciones, al poder evaluar el comportamiento de elementos aleantes como el cobre, níquel o titanio en pos de una mejora del comportamiento a la corrosión atmosférica marina de los aceros y fundiciones.

\section{AGRADECIMIENTOS}

Los autores desean agradecer a los proyectos DICYT, Código 051614AA, Vicerrectoría de Investigación, Desarrollo e Innovación, y al proyecto 1555LD ambos de la Universidad de Santiago.

Rodrigo Allende desea agradecer a Comisión Nacional de Investigación Científica y Tecnológica, CONICYT-PFCHA/Doctorado Nacional/2017-21170167 por el financiamiento de sus estudios de postgrado.

\section{BIBLIOGRAFÍA}

[1] VERA, R., DELGADO, D., ARAYA, R., et al., "Construcción de mapas de corrosión atmosférica de chile: Resultados preliminares", Revista Latinoamericana de Metalurgia y Materiales, v. 32, n. 2, pp. 269276, Nov. 2012.

[2] MORCILLO, M., CHICO, B., DÍAZ, I., et al., "Atmospheric corrosion data of weathering steels. A review”, Corrosion Science, v. 77, n.1, pp. 6-24, Sept. 2013.

[3] MORCILLO, M., CHICO, B., DÍAZ, I., et al., "Atmospheric corrosion of Ni-advanced weathering steels in marine atmospheres of moderate salinity”, Corrosion Science, v. 76, n. 1, pp. 348-360, Jul. 2013.

[4] SOUTHWELL, C., BULTMAN, J., ALEXANDER, A., "The corrosion rates of structural metals in seawater, fresh water and tropical atmospheres: Summary of a sixteen-year exposure study", Modern Casting, v. 19, n. 3, pp. 179-183, Mar. 1969. 
[5] SURNAM, B., CHUI, C., XIAO, H., et al., "Investigating atmospheric corrosion behavior of carbon steel in coastal regions of Mauritius using Raman Spectroscopy", Revista Matéria, v. 21, n. 1, pp. 157-168, Mar. 2016.

[6] OLAWALE, J., ODUSOTE, J., RABIU, A., et al., "Evaluation of Corrosion Behaviour of Grey Cast Iron and Low Alloy Steel in Cocoa Liquor and Well Water", Journal of Minerals and Materials Characterization and Engineering, v. 1, n. 2, pp. 44-48, Mar. 2013.

[7] ARTIGAS, A., MONSALVE, A., SIPOS, K., et al., "Development of accelerated wet-dry cycle corrosion test in marine environment for weathering steels", Corrosion Engineering, Science and Technology, v. 50, n. 8, pp. 628-632, Feb. 2015.

[8] ARTIGAS, A., BUSTOS, O., GARZA-MONTES-DE-OCA, N., et al., "Influencia de Ni y Ti en la respuesta a la corrosión marina simulada de un acero autopatinable A242", Revista Matéria, v. 20, n. 3, pp. 646652. Sept. 2015.

[9] ARTIGAS, A., BUSTOS, O., GARZA-MONTES-DE-OCA, N., et al., "Comportamiento a la corrosión atmosférica marina de aceros autopatinables con estructura ferrítico perlítica y ferrítico martensítica", Revista Matéria, v. 20, n. 3, pp. 659-667, Sep. 2015.

[10] WANG, J., WANG, Z., "Corrosion behaviour of weathering steel in diluted Qinghai saltlake water in a laboratory accelerated test that involved cyclic wet/dry conditions", Materials Chemistry and Physics, v. 124, n- 2-3, pp. 952-958, Dic. 2010.

[11] DUNN, D., BROSSIA, C., BOGART, M., et al., "Corrosion of Iron Under Alternating Wet and Dry Conditions", Corrosion 99, NACE-99223, San Antonio, Texas, USA, 25-30 Abril 1999.

[12] WANG, Z., YIN, F., WU, L., et al., "Corrosion Resistance on High Strength Bainitic Steel and 09cupcrni after Wet-Dry Cyclic Conditions", Journal of Iron and Steel Research, v. 20, n. 2, pp. 72-78, Feb. 2013.

[13] ZHANG, W., YANG, S., GUO, J., et al., "Incubation and development of corrosion in micro-estructures of low alloys steels under a thin liquid film of $\mathrm{NaCl}$ aqueous solution", International Journal of Minerals, Metallurgy, and Materials, v.17, n. 6, pp. 748-755, Dic. 2010.

[14] DONG, J., HAO, L., ZHANG, S., et al., "A study the evolution of rust on Mo-Cu-bearing fire-resistant steel submitted to simulated atmospheric corrosion", Corrosion Science, v. 54, n. 1, pp. 244-250, Ene. 2012.

[15] HAO, L., ZHANG, S., DONG, J., et al., "Evolution of atmospheric corrosion of MnCuP weathering steel in a simulated coastal-industrial atmosphere". Corrosion Science, v. 59, n. 1, pp. 270-276, Mar 2012.

[16] AKIYAMA, E., LI, S., SHINOHARA, T., ZHANG, Z., et al., "Hydrogen entry into Fe high strength steels under simulated atmospheric corrosion”, Electrochemical Acta., v. 56, n. 4, pp. 1799-1805, Ene. 2011.

[17] ASTM Standard A48/A48M - 03 (Reapproved 2012), 2012, " Standard Specification for Gray Iron Castings", ASTM International, West Conshohocken, PA, 10.1520/A0048_A0048M-03R12, http://www.astm.org.

[18] MELCHERS, R., JEFFREY, R., "The critical involvement of anaerobic bacterial activity in modelling the corrosion behaviour of mild steel in marine environments", Electrochimica Acta, v. 54, n. 1, pp. 80-85, Mar. 2008.

[19] MELCHERS, R., "Microbiological and abiotic processes in modelling longer-term marine corrosion of steel", Bioelectrochemistry, v. 97, n. 1, pp. 186-194, Jun. 2014.

[20] MELCHERS, R.," Modeling of Marine Corrosion of Steel Specimens", Corrosion Testing in Natural Waters, ASTM STP 1300, Philadelphia, Pensilvania, USA, 1 Abril 1997.

[21] MELCHERS, R., "Long-term corrosion of cast irons and steel in marine and atmospheric environments", Corrosion Science, v. 68, n. 1, pp. 186-194, Mar. 2013.

[22] ASTM Standard B117 - 16, 2016, "Standard Practice for Operating Salt Spray (Fog) Apparatus", ASTM International, West Conshohocken, PA, DOI: 10.1520/B0117-16, http://www.astm.org.

[23] KIMURA, T., NASU, S., TAZAKI, T., et al., "Mossbauer spectroscopic study of rust formed on a weathering steel and a mild steel exposed for a long term in an industrial environment", Materials Transactions, v. 43, pp. 694-703, Feb. 2002.

[24] BADAN, B., MAGRINI, M., RAMOUS, E., "A study of the microbiological-corrosion products of steel and cast iron pipes in fresh water", Journal of Materials Science, v. 27, n. 1, pp. 1951-1954, Ene. 1991. 
[25] MOHEBBI, H., LI, C. Q., "Experimental Investigation on Corrosion of Cast Iron Pipes”, International Journal of Corrosion, v. 1, n. 1, Mar. 2011.

[26] ASTM Standard G1 - 03 (Reapproved 2011), 2012, " Standard Practice for Preparing, Cleaning, and Evaluating Corrosion Test Specimens", ASTM International, West Conshohocken, PA, DOI: 10.1520/G0001-03R11, http://www.astm.org.

[27] RAO, B., KUMAR, P., RAO, S., JAGDISHCHANDRA K., et al., "Bactericidal Effect of Direct and Filtered Ultraviolet C Through Transparent Plastic Sheet on Gram Negative Bacilli -An In- Vitro Study", International Journal of Biomedical and Advance Research, v. 3, n. 10, pp. 785-790, 2012.

[28] ALBRECHT, P., HALL, T., JR. "Atmospheric Corrosion Resistance of Structural Steels", Journal of Materials in Civil Engineering., v. 12, n. 1, pp. 2-24, Feb. 2003.

[29] MOHEBBI, H., LI, C. "Experimental Investigation on Corrosion of Cast Iron Pipes", International Journal of Corrosion, v. 2011, n. 2011, pp. 1-17, Mar. 2011.

[30] KIMURA, M., KIHIRA, H., OHTA N., et al., "Control of $\mathrm{Fe}(\mathrm{O}, \mathrm{OH})_{6}$ nano-network structures of rust for high atmospheric-corrosion resistance", Corrosion Science., v. 47, n. 10, pp. 2499-2509, Jun. 2005.

[31] ANDERKO A., SHULER P., "Computational approach to predicting the formation of iro2n sulphide species using stability diagrams", Computers \& Geosciences, v. 23, n.6, pp. 647-658, Jul. 1997.

[32] NEAL, A., TECHKARNJANARUK, S., DOHNALKOVA, A., et al., "Iron sulfides and sulfur species produced at hematite surfaces in the presence of sulfate-reducing bacteria", Geochimica et Cosmochimica Acta, v. 65, n. 2, pp. 223-235, Ene. 2001.

[33] MELCHERS, R., JEFFREY, R. "Bacteriological influence in the development of iron sulphide species in marine immersion environments", Corrosion Science, v. 45, n. 4, pp. 693-714, Abr. 2003. 